\title{
PLAN MAESTRO HUMEDALES
}

Dirección Institucional: Mgter. Arq. Fabián Echezarreta. Dirección General: Mgter. Arq. Lorena Cesira Sánchez; Mgter. Arq. Julieta Repetto. Coordinación Operativa: Lic. Laura Andrea Cochatok Díaz.

Equipo de consultores: dirección, Arq. Marta Aguilar; coordinación general, Lic. Daniela Tregierman;

equipo de profesionales, Mgter. Prof. Jorge Blanco, Arq. Yanina Antonelli, Lic. Luis Baruzzo,

Arq. Clarisa Castella, Lic. Patricia Cirillo, Ing. Carlos Guardia, Arq. Diana Levraux, Ab. Anahí Lindström,

Lic. Norma López, Ing. Mariano Molina, Arq. Fernanda Pesado, Ing. Mariela Quiroga,

Arq. Marcelo Scarfó, Lic. Corina Velárdez, Arq. Carlos Verdecchia.

Consultora: Viator Proyectos SA, Arq. Carlos Pisoni.

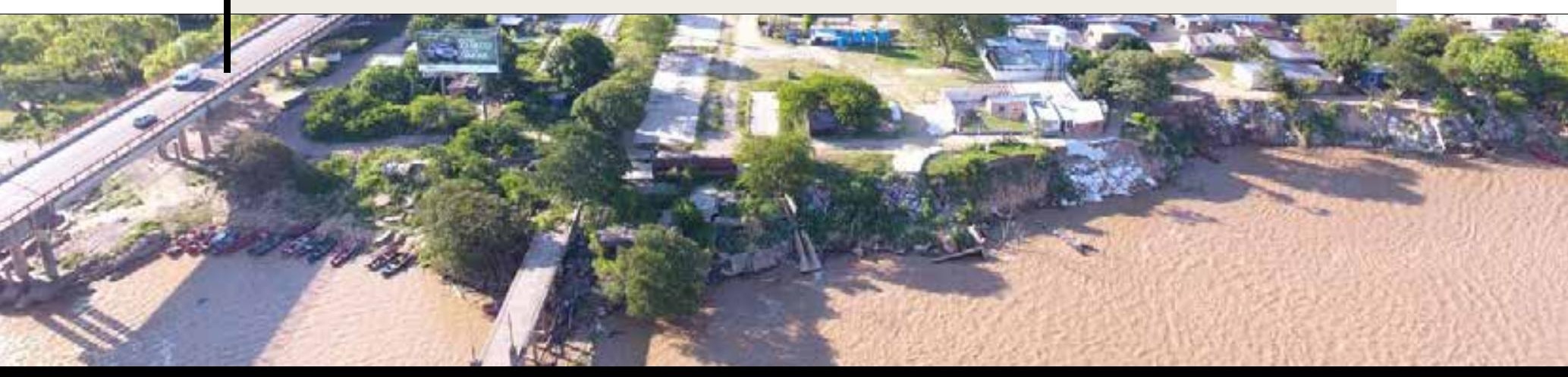




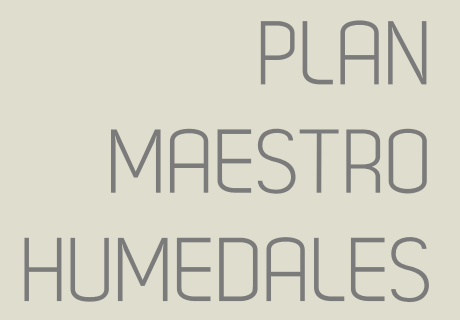

\section{PALABRAS CLAUE}

Planificación territorial; Plan Maestro Humedales; Chaco.

\section{RESUMEN}

El Plan Maestro Humedales forma parte del grupo de iniciativas regionales que la gestión provincial ha priorizado en materia de planificación integral para la provincia, y tiene por objetivo aportar al gobierno un instrumento para la gestión integral del territorio que colabore en el fortalecimiento de su accionar en relación con la conservación y gestión sustentables del Humedal Chaco. Este trabajo fue desarrollado en el marco de un convenio entre el gobierno provincial con el Consejo Federal de Inversiones (CFI) y Viator Proyectos $\mathrm{SA}$, con la coordinación general de la Subsecretaría de Planificación y Proyectos del Ministerio de Infraestructura y Servicios Públicos.

\section{KEYWORDS}

Territorial planning, master plan wetlands, Chaco.

\section{ABSTRACT}

The Master Plan Wetlands is part of the group of regional initiatives that the provincial government has prioritized in the matter of integral planning for the Province. Its aim is to provide the government with an instrument for the integral management of the territory that collaborate in strengthening its actions in relation to the sustainable conservation and management of the Chaco Wetland. This work was developed within the frame of an agreement between the provincial government with the Federal Investment Council and VIATOR Proyectos S. A., under the general coordination of the Under-secretariat of Planning and Projects of the Ministry of Infrastructure and Public Services. 


\section{El plan en el marco de la gestión de gobierno de la provincia del Chaco}

El Plan Maestro Humedales forma parte del grupo de iniciativas regionales que la gestión provincial ha priorizado en materia de planificación integral para la provincia. Este grupo, formado por tres regiones de gran importancia por sus características naturales, productivas y de concentración de población, actuará como ordenador de las políticas públicas provinciales. Ellos son el Plan Maestro Ciudad Río, que abarca las localidades por las que atraviesa el río Negro en su tramo regulado; el Máster Plan Impenetrable, que se extiende a lo largo de la zona norte de la provincia abarcando los municipios del Departamento General Güemes y el Municipio de Juan José Castelli, y finalmente el Plan Maestro Humedales.

Este último se desarrolla en este contexto, con el objeto de aportar al gobierno provincial un instrumento para la gestión integral del territorio que colabore en el fortalecimiento de su accionar en relación con la conservación y gestión sustentables del Humedal Chaco y su biodiversidad, que apunte a potenciar las características productivas y turísticas del lugar garantizando la sustentabilidad del ecosistema junto con la mejora de los indicadores y condiciones socio-territoriales de la población residente.
Con ese objetivo en convenio con el Consejo Federal de Inversiones (CFI) y de Viator Proyectos SA, se conformó un equipo de profesionales de diversas disciplinas con consultores locales y externos, que con la coordinación general de la Subsecretaría de Planificación y Proyectos del Ministerio de Infraestructura y Servicios Públicos llevó adelante el estudio integral del territorio. Se conformaron mesas de trabajo con actores institucionales, tanto de las diversas áreas de gobierno de la provincia como de cada uno de los municipios que conforman la región de los humedales, en cuyo marco se expusieron y validaron los principales problemas y oportunidades del humedal y se compartieron las iniciativas sectoriales existentes para este territorio.

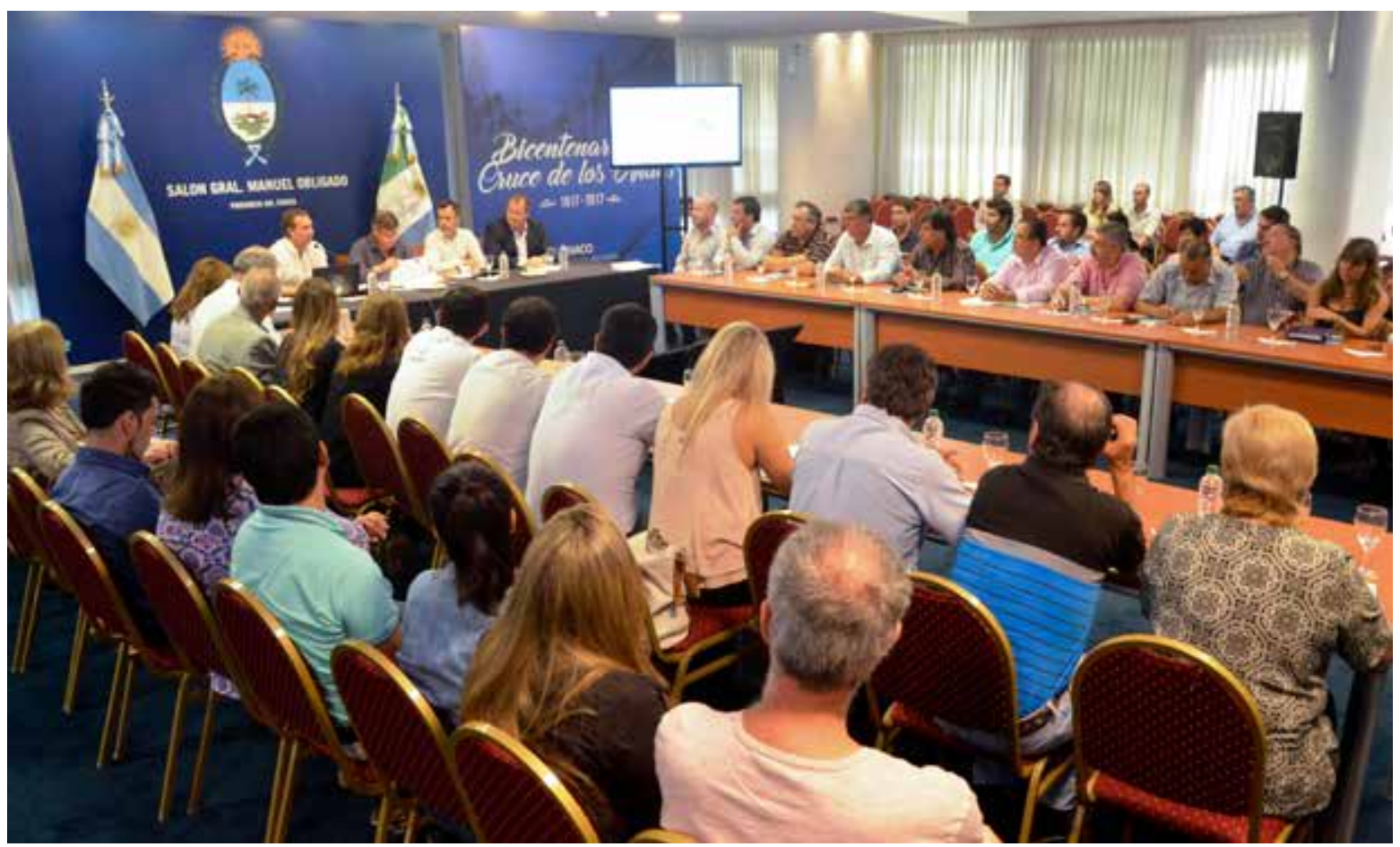

Mesa interinstitucional e intermunicipal. Presentación de avances del plan maestro 
Se propuso llevar adelante la metodología de planificación-acción, realizando reuniones en los municipios, recorriendo cada uno de ellos y fundamentalmente acompañándolos con intervenciones concretas tendientes a promocionary fortalecer las características identificadas como potenciales en cada localidad.

Producto de ese proceso, el Plan Maestro Humedales expresa los lineamientos de la política pública establecidos por la actual gestión del gobierno de Chaco y define una serie de estrategias singulares para el desarrollo sustentable en orden a sus características naturales, culturales y socioeconómicas, y propone un abanico de acciones que van desde las obras de pequeña escala - paseos, miradores, parques - a obras estratégicas de logística como puertos, puentes, rutas y energía, que potencian el desarrollo productivo de la provincia.

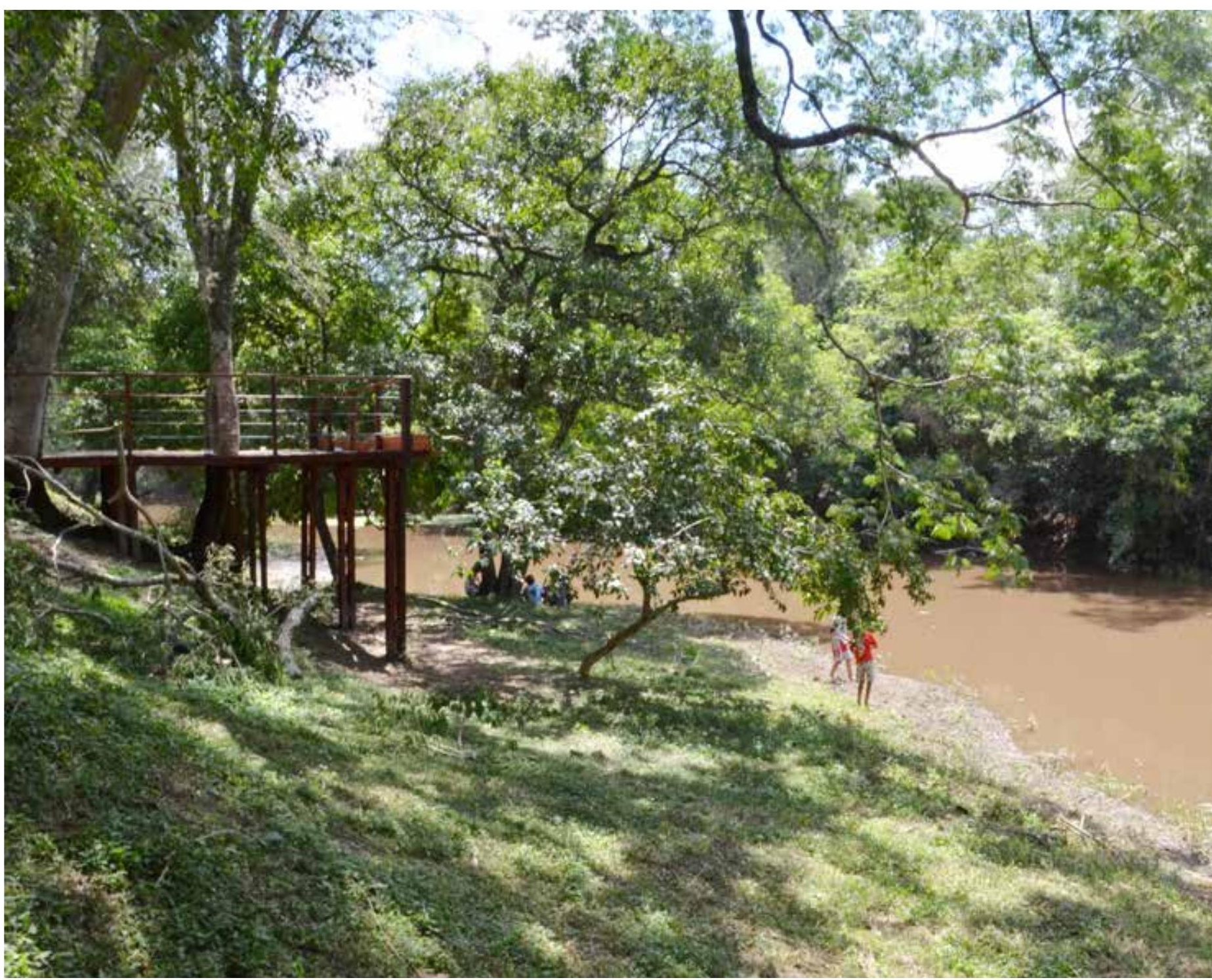

Recuperación del riacho Canguí 
2. Plan Maestro Humedales: contextualización ubicación geográfica, improntas de la región

En primer lugar, resulta importante mencionar que para el desarrollo de este plan se tuvieron en cuenta estudios previos, de los cuales el Plan Estratégico Territorial Chaco (PET Chaco, 2013) fue el antecedente fundamental que definió la orientación de las propuestas del plan maestro al aportar una visión integral del territorio en el entorno provincial y regional del humedal. El estudio abarca en su totalidad la zona de humedales de la provincia del Chaco, declarada en 2004 como sitio Ramsar de importancia a nivel internacional. Protegido particularmente por su valor medioambiental en el marco de dicha convención internacional, constituye uno de los ecosistemas de mayor diversidad biológica de la Argentina y alcanza un área aproximada de un millón de hectáreas. Concentra además a casi el $40 \%$ de la población total y atraviesa la capital provincial y los puntos estratégicos de conexión regional e internacional

Está ubicado en la franja oriental de los departamentos San Fernando, 1. ${ }^{\circ}$ de Mayo y Bermejo y presenta los siguientes límites: sur, que coincide con el paralelo 28, que marca la frontera con la provincia de Santa $\mathrm{Fe}$; límite norte con el cauce del río Bermejo; límite oeste con la Ruta Nacional N. ${ }^{0} 11$ y límite este con los cauces de los ríos Paraná y Paraguay. Comprende un total de doce municipios, con una población que alcanzaba los 394.221 habitantes, según el censo de 2010. Dicha pobla- ción se distribuye en un sistema de asentamientos de diversa jerarquía - rurales y urbanos -, entre los que se encuentra el Área Metropolitana del Gran Resistencia, ciudad capital de la provincia

La población se distribuye en las tres formas de asentamiento: urbana rural agrupada y rural dispersa. La población urbana y rural agrupada representa el 98 \% del total del área. Esta caracterización da cuenta de uno de los principales desafíos que comporta este sector del territorio provincial, en tanto coexisten en él un valoradoecosistemaquepreservarcon un alto grado de ocupación producto del proceso histórico de asentamiento poblacional en la provincia.

La provincia del Chaco se halla integramente en el sector denominado Chaco Austral y posee un relieve totalmente llano y aluvial, con una muy leve inclinación en sentido NO-SE. Esta característica se hace evidente en la dirección de los cursos fluviales muchas veces divagantes. Los suelos son mayoritariamente arcillosos, lo que sumado a la escasa pendiente dificulta el escurrimiento de las aguas y forma numerosos bañados, esteros y lagunas de carácter semipermanente. De este modo, el escurrimiento está regido por la morfología regional y condicionado por los rasgos climáticos que, sumados a la baja energía del relieve, determinan el modelo de escurrimiento lento y complejo, de tipo laminar, con escasa capacidad de evacuación en los períodos de precipitaciones ordinarias.

En lo que respecta a nuestra área de estudio, estas características se agudizan en directa relación con la dinámica territorial de las distintas subregiones del corredor fluvial Paraná-Paraguay, del que forma parte. Durante los ciclos de precipitaciones extraordinarias o en períodos húmedos, el área en su mayor parte se convierte en un ambiente con distintos grados de anegabilidad, con una lámina de agua continua o discontinua. Esta combinación de rasgos climáticos, geomorfológicos e hídricos permite identificar zonas con distintas restricciones al uso del suelo, caracterizadas por sus cotasy por la recurrencia de inundaciones.

\section{Puede afirmarse, por tanto, que la unidad territorial que compone el Humedal Chacoestá determinada por el imperativo hídrico que le otorga identidad y valor como ecosistema, al mismotiempoque define su devenir al contener un abanico de situaciones ambien- tales y territoriales explicativas de su patrón de ocupación, así como de su dinámica poblacional y productiva.}

En función de ello, se identifican en el territorio tres sectores claramente diferenciados: el humedal sur, el humedal centro y el humedal norte. En esta diferenciación cumple un rol fundamental la mayor aglomeración de población provincial, el Área Metropolitana de Resistencia, que da entidad al sector central y que fragmenta y articula al mismo tiempo la continuidad de los humedales hacia el sur y hacia el norte.

El humedal sur se extiende desde el límite con la provincia de Santa Fe hasta la ciudad de Resistencia. La mayor parte del área se encuentra dentro de la terraza de inundación del río Paraná, que tiene en este sector un ancho considerable, que hace que quede prácticamente toda 


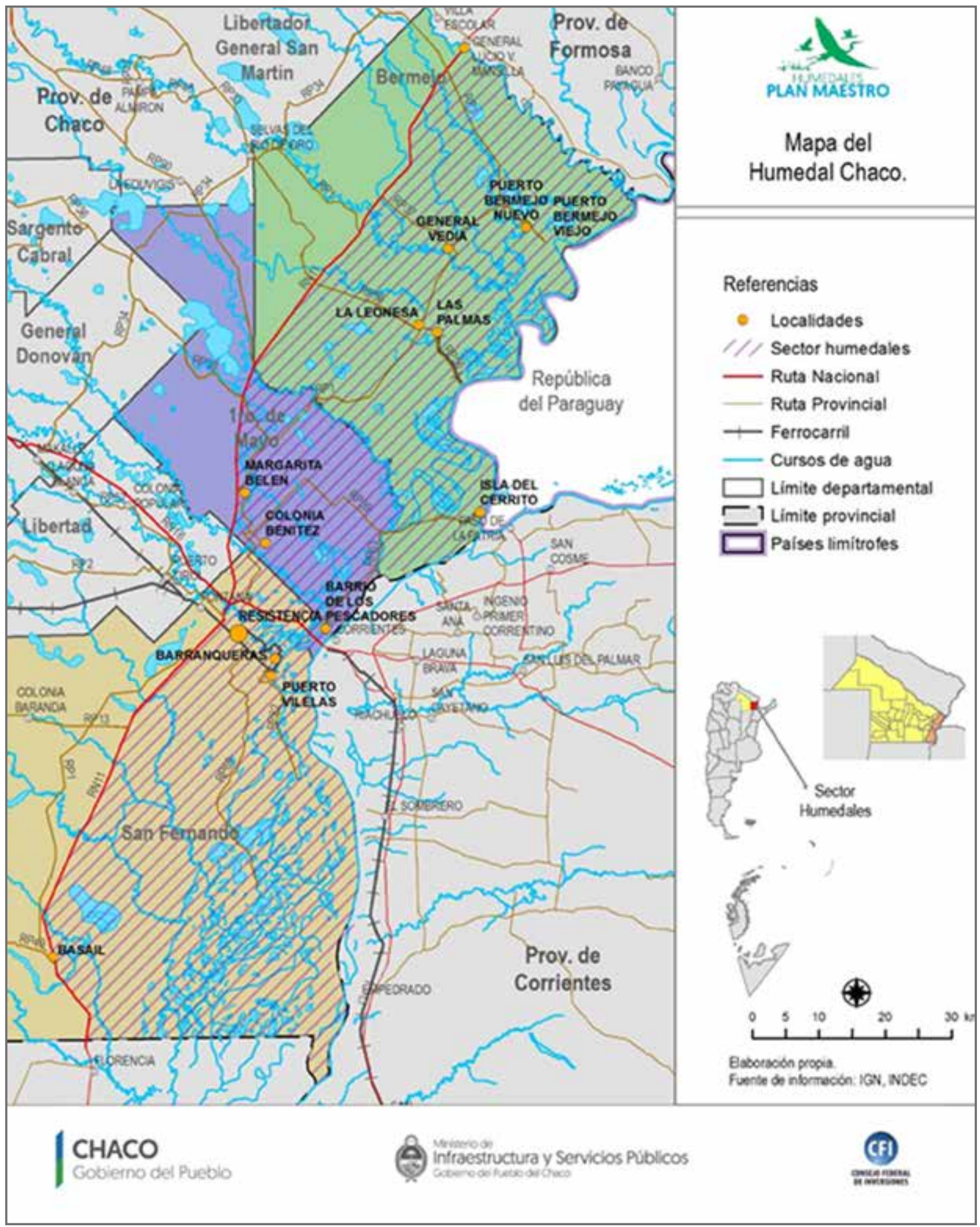




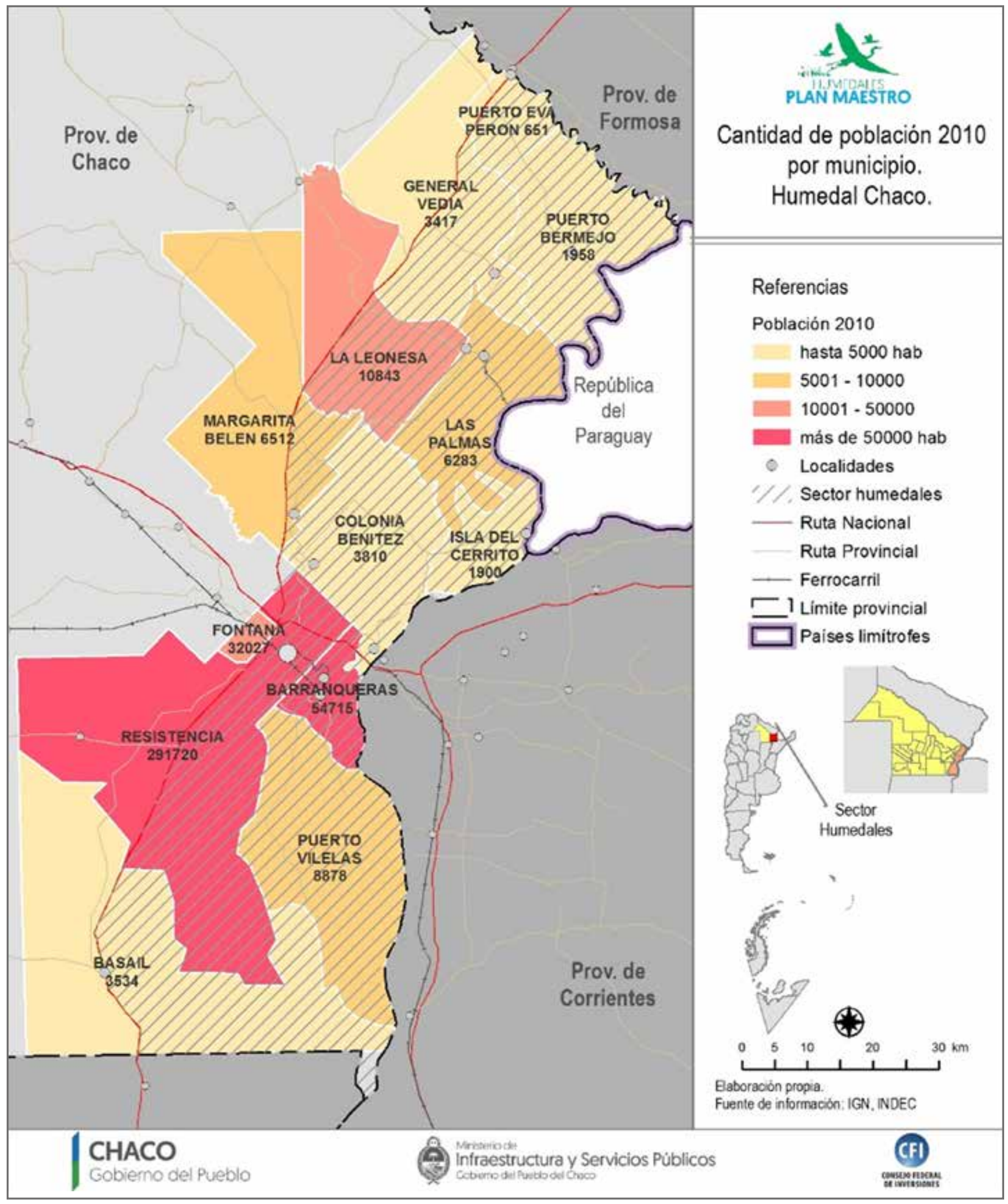




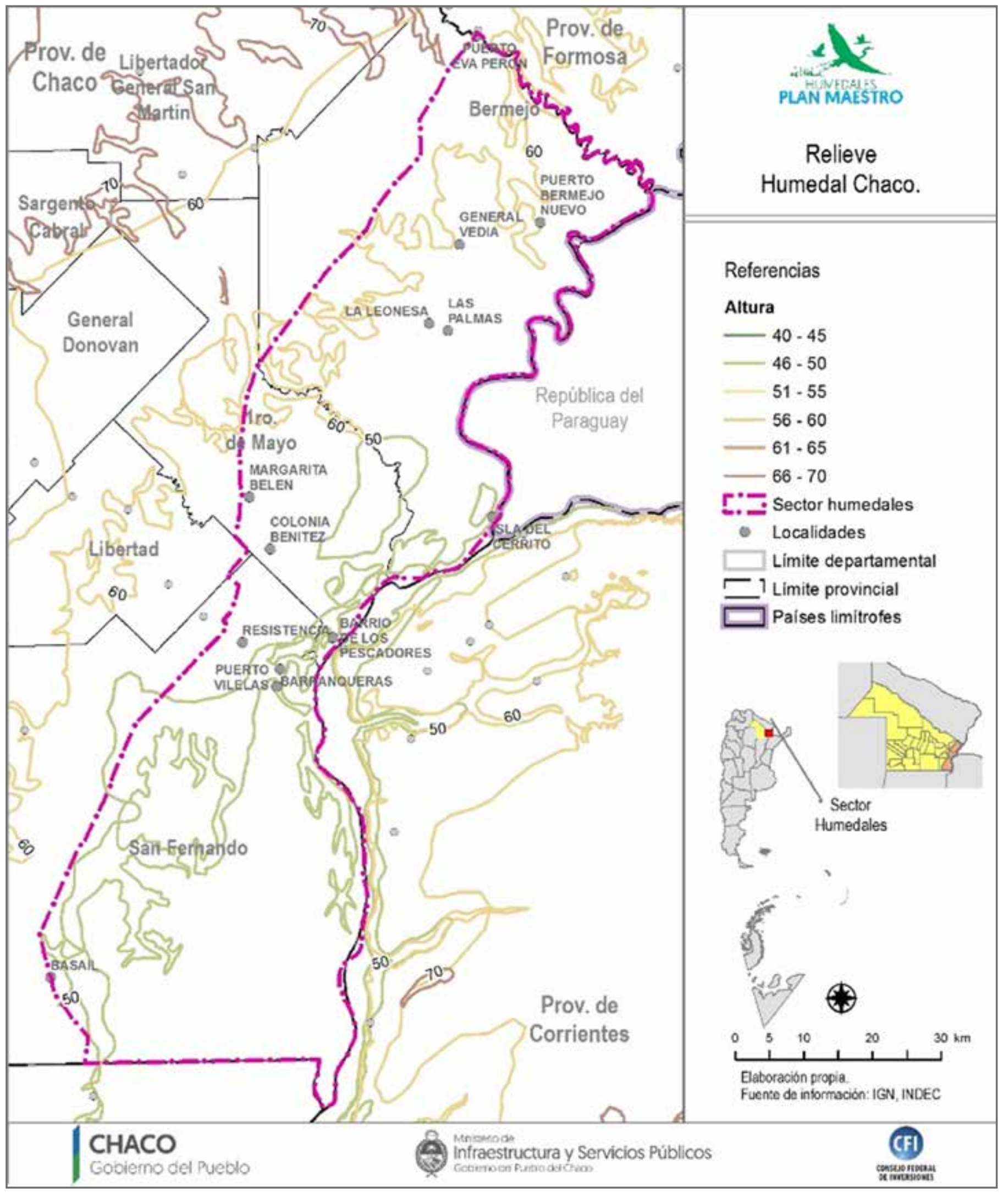




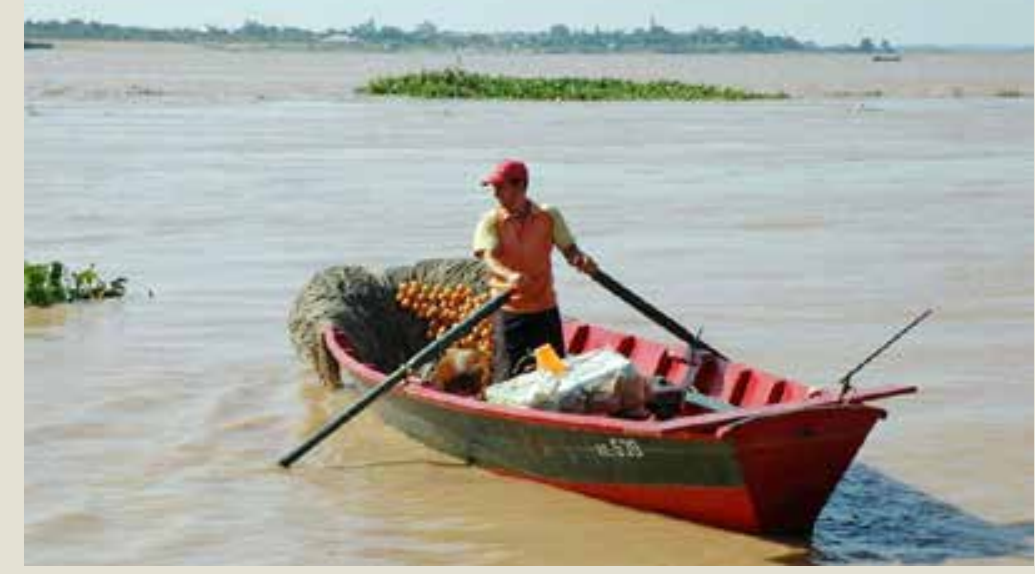

su superficie dividida en dos áreas, de restricción hídrica severa y leve, respectivamente.

El humedal centro se define por el intenso proceso de antropización, representado por el conjunto de localidades que conforman el AMGR, principal concentración poblacional de la provincia, con diversidad de equipamiento y una compleja infraestructura, la cual ocupa un lugar relevante en el nivel nacional, potenciado por la vinculación con la ciudad de Corrientes. Las actividades económicas del humedal centro tienen la impronta propia de los grandes centros urbanos, destacándose los diferentes tipos de servicios administrativos, culturales, educativos, de salud, comerciales, etc., que provee el núcleo Resistencia, así como su función como nodo de articulación de infraestructura de circulación diversificada y multiescalar.

El humedal norte se extiende desde la ciudad de Resistencia hasta el límite con la provincia de Formosa, y solo una estrecha franja del área se encuentra dentro del valle de inundación del río Paraguay, con restricción hídrica severa o leve. Es decir que, en toda la franja nordeste del territorio, las condiciones hídricas son menos determinantes y hay amplias porciones de suelo con capacidad productiva de diverso tipo.

En este sector se asienta la mayor parte de la población del humedal, con excepción del AMGR, reuniendo en total más de 25.000 habitantes entre población urbana y rural. Se destacan, en primer lugar, las localidades de La Leonesa y Las Palmas, que conforman entre ellas un núcleo de centralidad de referencia en el sector, ya que suman 17.000 habitantes. La Leonesa es cabecera del departamento Bermejo y reúne una importante cantidad y variedad de equipamientos de servicios para la población local: escuelas, centros de salud, banco. Las Palmas, por su parte, a pesar de haber perdido población en el último período intercensal, está vinculada con el crecimiento de la actividad arrocera combinada con la cría de pacú. Las caracteristicas de ambas localidades dan cuenta de la necesidad de un abordaje integrado.

En la zona se asientan, además, otras localidades con rango municipal, como General Vedia, Puerto Eva Perón y Puerto Bermejo, que aportan 6000 habitantes, vinculadas con el desarrollo de actividades productivas de escala familiar con alto potencial de crecimiento y diversificación. Finalmente, se destaca en esta área el municipio de Isla del Cerrito, con cerca de 2000 habitantes, una localización privilegiada en términos de calidad ambiental y paisajística, con unacervo edilicio patrimonial histórico y con vocación como polo turístico regional, basado en la existencia de equipamientos recuperables y una larga tradición cultural y recreativa alrededor de la pesca del dorado, y articulada principalmenteconelnúcleo del AMGR. Las principales actividades del humedal norte son, como ya se mencionó, la producción de arroz, asociada con la piscicultura, la ganadería, la agricultura desarrollada por pequeños productores y la actividad industrial de procesamiento de pacú.

Las características diferenciadas de estos tres sectores del Humedal Chaco dan cuenta de la pertinencia de atenderlos como subsistemas, debido a las condiciones que imponen tanto la dinámica ambiental como el medio construido para el establecimiento de estrategias de desarrollo integrales.

\section{Principales nudos problemáticos}

El área de humedales del Chaco se presenta como un territorio complejo, con particularidades significativas que constituyen la base conceptual para la formulación de las propuestas del plan maestro. En primer lugar, el propio carácter de humedal impone una impronta distintiva: el reconocimiento de las múltiples funciones y servicios que presta el ecosistema y la necesidad de respetar ese "imperativo hídrico". El humedal establece, además, una temporalidad propia, un ritmo particular en el paisaje, que debe ser incorporado en las propuestas de manejo. En segundo lugar, el diagnóstico del área de humedales da cuenta de una situación social en el área que requiere acciones concretas para mejorar las condiciones de vida de la población, y se genera asi un segundo "imperativo": el de propender a la equidad social como eje de las propuestas del plan maestro. La caracterización de los núcleos problemáticos que se detallan a continuación tiene estos dos imperativos como guía para su formulación.

\section{A. Lagestión integraldelhumedal} El primer núcleo problemático se constituyeentornoalagestiónintegral del humedal. El diagnóstico provee una identificación de cuestiones que tienen que ser abordadas desde esa perspectiva, y entre ellas se destaca la necesidad de asegurar las funciones y los servicios del humedal con la periodicidad que caracteriza su dinámica hídrica. Además, el carácter de sitio Ramsar da cuenta de sus singularidades, al tiempo que compromete la formulación de un 
plan de manejo integral.

\section{B. El desafío del}

\section{desarrollo sostenible}

El segundo núcleo problemático está definido en la tensión entre el desarrollo de la producción y las condiciones ambientales del humedal, articulados en la idea de desarrollo sostenible, con lo cual es necesaria la atención de conflictos entre las dinámicas de la producción y las condiciones de vida de los pequeños productores, que mejore en términos generales las condiciones de vida de la población.

\section{La integración territorial: conectividad}

Eldiagnóstico del territoriohumedales recoge diversos elementos que dan cuenta de la conectividad como uno de los núcleos problemáticos, ya que en el caso particular se trata de una red vial que en su conjunto requiere un cuidado estructural y un mantenimiento permanente para adaptarse a las condiciones climáticas y a los pulsos del humedal, que pueden comprometer seriamente la disponibilidad de estas vías de circulación. Pero la movilidad no se concreta solamente con la presencia de infraestructura, sino que debe considerar también la oferta de servicios de transporte. En este punto, el núcleo problemático vinculado con la conectividad exige también una consideración de alternativas para las redes de servicios de transporte, que amplien la cobertura, mejoren las frecuencias y faciliten la conectividad interna microrregional para atender el variado abanico de necesidades de desplazamiento de los habitantes del humedal.

\section{La articulación del} sistema de centros

Desde el punto de vista de la organización del sistema urbano, el hecho más destacado - y que constituye una fortaleza para el área- es la cercanía delnodo Resistencia-Corrientes, centro de servicios con una oferta complejay diversificada con importancia regional.
Como se describe, este nodo agrupa una serie de localidades con una vinculación física y funcional muy estrecha, entre las que se encuentran la propia Resistencia, Barranqueras, Fontanay Vilelas. Estenúcleo polariza, en el nivel microrregional, algunos centros urbanos pequeños situados a corta distancia, como Margarita Belén, Colonia Benítez y barrio Pedro Pescador, mientras que el sector sur del humedal, por su parte, solo cuenta con el pequeño núcleo de población rural agrupada de Basail.

En el norte, en cambio, hay un grupo de localidades que aún no consiguen estructurar un subsistema con capacidad de servicios para atender a toda la población del área, tanto urbana como rural. El asentamiento más importante es el formado por La Leonesa y Las Palmas, con potencial para desarrollar aún más una centralidad a escala microrregional, con equipamientos y oferta de servicios para todo el norte del humedal.

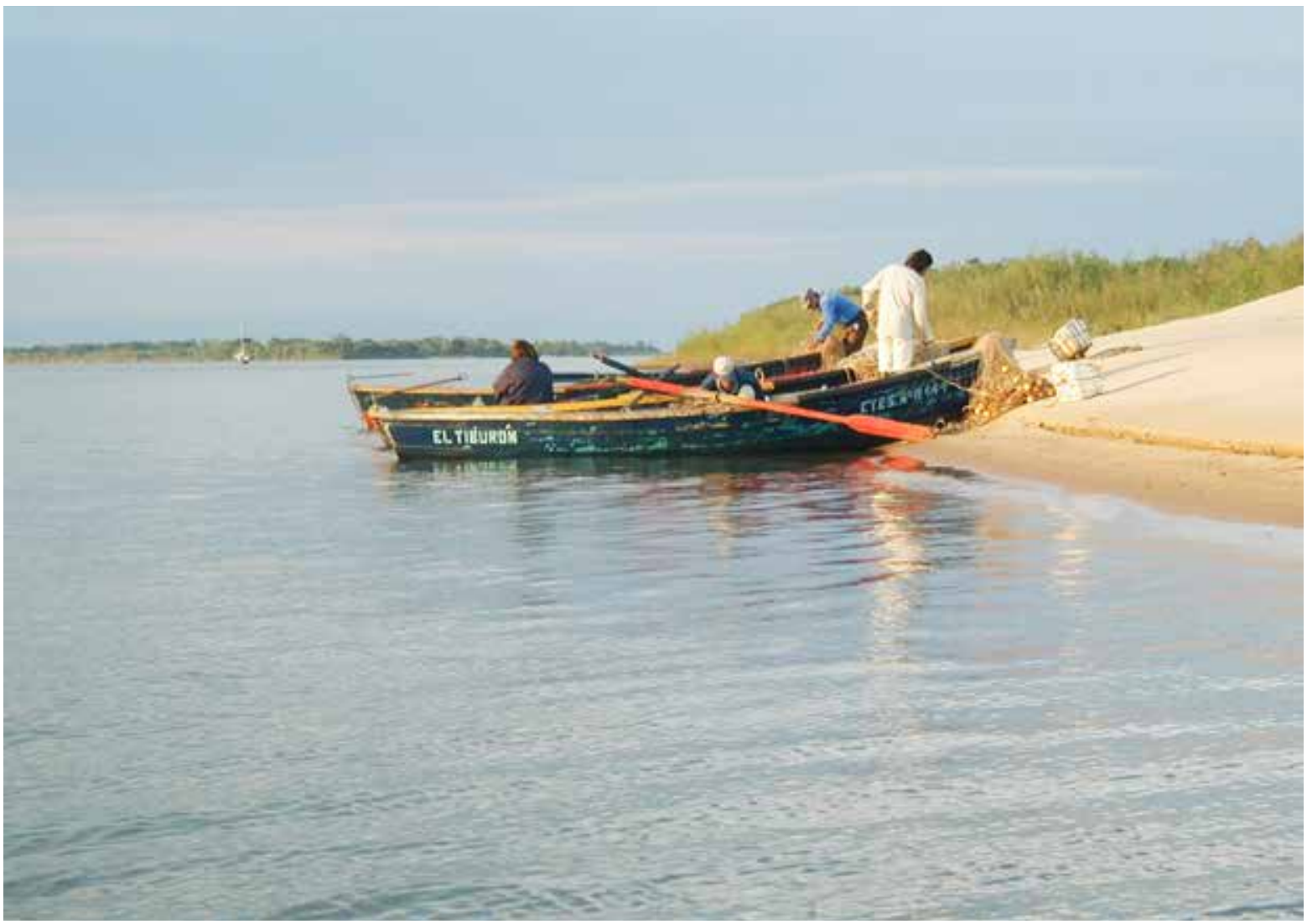

Actividad mallonera. Río Paraná 


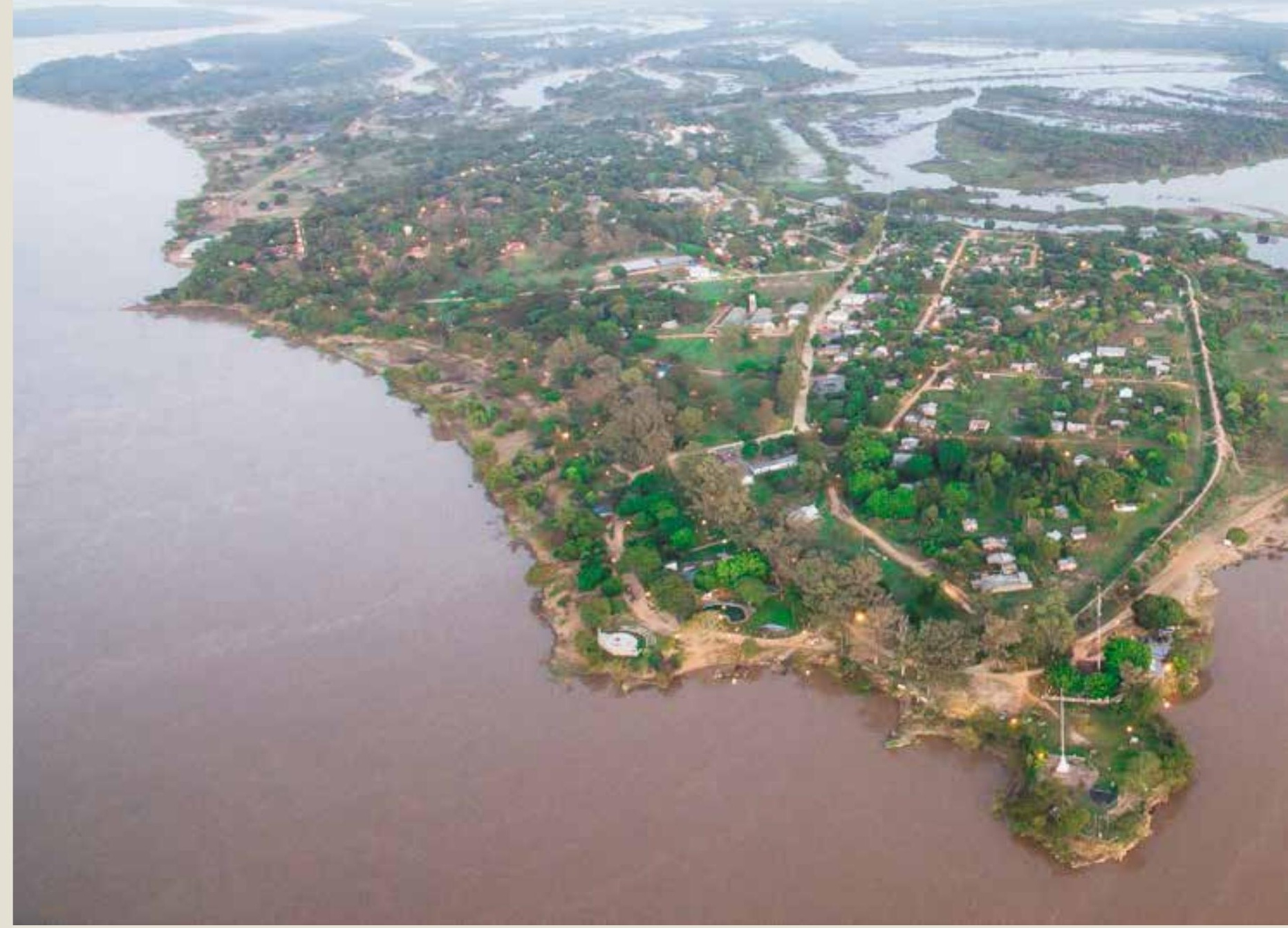

Imagen aérea de la Isla del Cerrito. Foto: Juan Ignacio Fuentes.

\section{E. El abordaje de los conflictos dominiales y del crecimiento urbano}

Es importante considerar que, si bien los porcentajes de crecimiento de la población no son demasiado elevados, la formación de nuevos hogares sigue un ritmo superior, yes esta unidad, el hogar, el demandante de nuevas viviendas y servicios. Asimismo, cabe prestar atención a otras dos tensiones: la que se genera entre ocupación del suelo y áreas inundables siguiendo el ritmo del humedalyel potencial conflicto entre usos en la interfase urbano-rural.

Por otro lado, si bien la población rural dispersa se reduce en términos absolutos, también se han identificado en el ámbito rural problemas de tenencia del sueloy de condiciones de las viviendas. Se desprenden, en consecuencia, algunos desafíos: explorar estrategias para garantizar el acceso al suelo urbano, ordenar el crecimiento de las localidades y prever la expansión futura.

\section{F. La gestión del riesgo y la reducción de la vulnerabilidad} La gestión del riesgo se basa en el reconocimiento de las amenazas y las vulnerabilidades. Las amenazas provienen de la dinámica hídrica del sector de humedales, sujeto a inundaciones frecuentes, mientras que las vulnerabilidades derivan de los procesos de construcción material del territorio: formas de ocupación del suelo, condiciones de las viviendas, características de la infraestructura de circulación y de los equipamientos. La gestión del riesgo incluye, como dimensión clave, la institucionalidad que se organiza en torno a los diferentes momentos asociados con el riesgo: la previsión, la emergencia, la pos emergencia y el largo plazo, y entonces, adicionalmente, es una situación que pone en juego diferentes escalas territoriales y diferentes actores institucionales. 


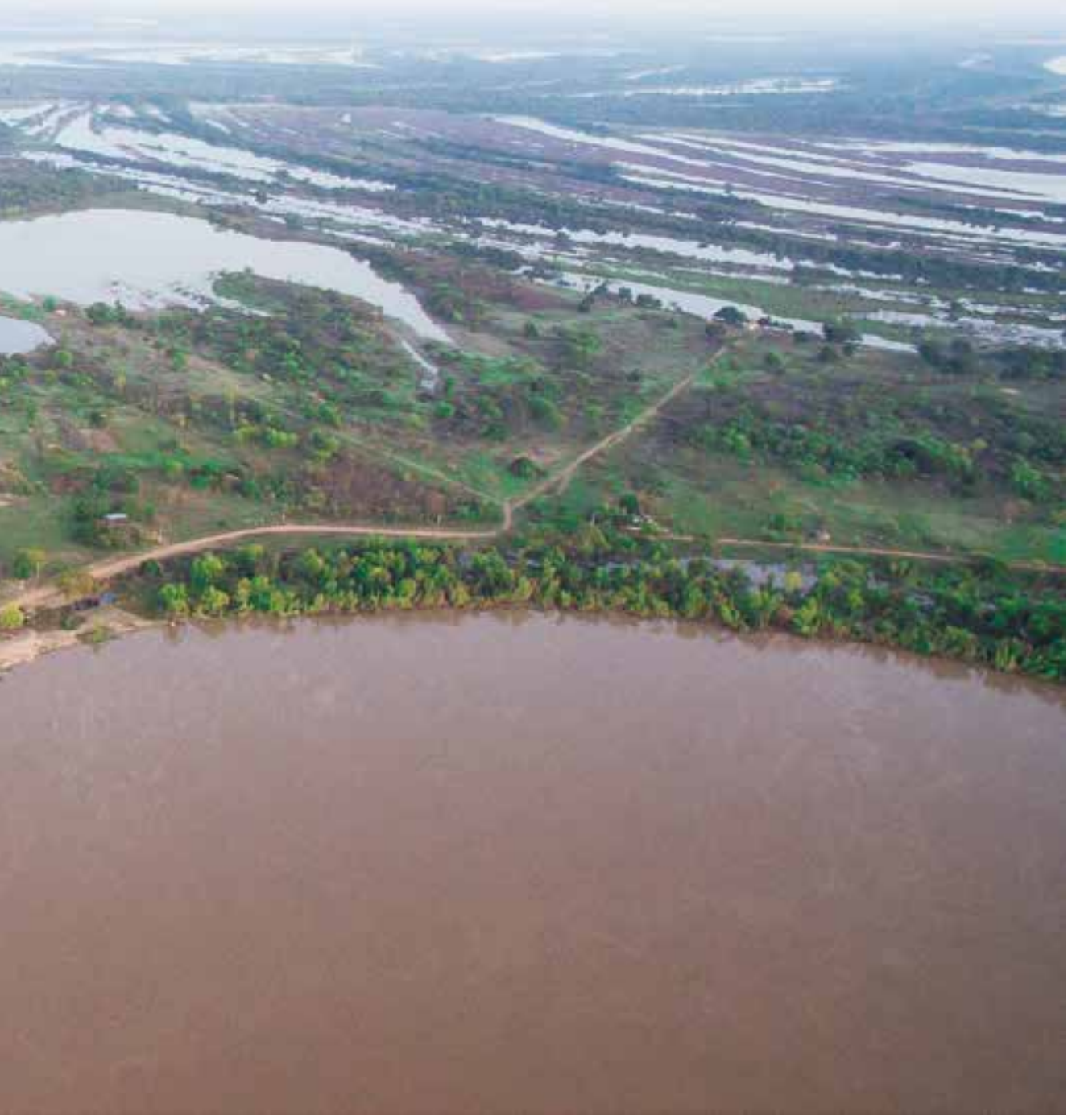

aproximación integral al humedal, que supere las visiones fragmentadas sectoriales y territoriales.

\section{Propuesta estratégica para el desarrollo sustentable}

La definición de un plan maestro, entendido como instrumento de gobierno, requiere la identificacióny combinación virtuosa de iniciativas de naturaleza concurrente: las de inversión en obras con aquellas de fortalecimiento social e institucional. Para encaminar el logro de sus objetivos, estas iniciativas deben conjugarse en el marco de una estrategia político-institucional que motorice el accionar de los organismos sectoriales e interjurisdiccionales que gravitan en el territorio. También con esta perspectiva integral ha madurado la fase propositiva del presente plan, traduciendo las problemáticas, oportunidades y desafíos territoriales en lineamientos estratégicos que enmarcan políticas sectoriales particularizadas.

La propuesta del Plan Maestro Humedales se organiza sobre la base de tres grandes ejes estratégicos que conjugan a su vez diversos programas de actuación territorial, a saber:

\section{G. La coordinación interinstitucional}

La dimensión institucional es crítica en la formulación del plan, en varios aspectos diferentes. En primer lugar, es una oportunidad para avanzar en la articulación de las numerosas y diversas iniciativas existentes, que incluyen programas y proyectos de escala nacional y provincial, como se presenta en este documento. En consecuencia, la iniciativa de la provincia en este sentido se ha encuadrado en una mesa interministerial que permitió recuperar visiones complementarias sobre los humedales del Chaco que fortalecieron el diagnóstico. La mirada desde la provincia promueve una
ESTRATEGIA 1:

Promoción del Desarrollo Económico-Productivo Inclusivo y Sustentable

ESTRATEGIA 2:

Inversión en Infraestructura, Equipamientos y Hábitat Construido

\section{ESTRATEGIA 3:}

Fortalecimiento Social e Institucional para el Gobierno del Territorio

El significado y contenido respectivo de las anteriores estrategias se explicita a continuación mediante dos instrumentos que son complementarios. 
El Modelo Deseado del Territorio, esquema sintético que representa espacialmente la visión de futuro o imagen objetivo que se propone para Humedal Chaco, y su expresión en la Matriz de Estrategias, Programas y Proyectos, que sintetiza y vincula las políticas y proyectos de intervención propuestos para encaminar las transformaciones territoriales buscadas.

\section{Modelo deseado del territorio}

El modelo reconoce como punto de partida las condiciones ambientales y productivas claramente diferenciadas de los tres sectores del Humedal Cha$\mathrm{co}$, condiciones de las que deviene su respectiva vocación territorial.

Un sector central decididamente antropizado, cuya vocación es sostener y consolidar su rol de nodo regional del nordeste, en estrecha vinculación con la ciudad de Corrientes. La impronta que hoy impone la red de infraestructuras que confluyen en el sector - provincial, nacional y regional- será aún más fuerte cuando se logre la construcción de las obras propuestas. Estas constituyen una tríada que tiende a fortalecer el papel del AMGR como polo logístico y de servicios regional. Se trata del segundo puente Chaco-Corrientes, de carácter ferrovial, proyectado en una ubicación próxima al eje que componen Puerto Vilelas-Riachuelo, de las provincias de Chaco y Corrientes, respectivamente; la puesta en operación del Ferrocarril Belgrano Norte, pieza fundamental del Plan Belgrano comprometido por el actual gobierno nacional y componente del eje de integración sudamericana que vinculará los puertos del Atlántico y el Pacífico y las obras de consolidación operativa y mejora de la accesibilidad del puerto de Barranqueras.

En el sector sur, por el contrario, se encuentra el espacio geográfico menos intervenido, donde el humedal se expresa con toda su potencia y biodiversidad. El imperativo hídrico impone difíciles condiciones para el asentamiento humano y admite a la ganadería como actividad predominante.

\section{Modelo territorial deseado}

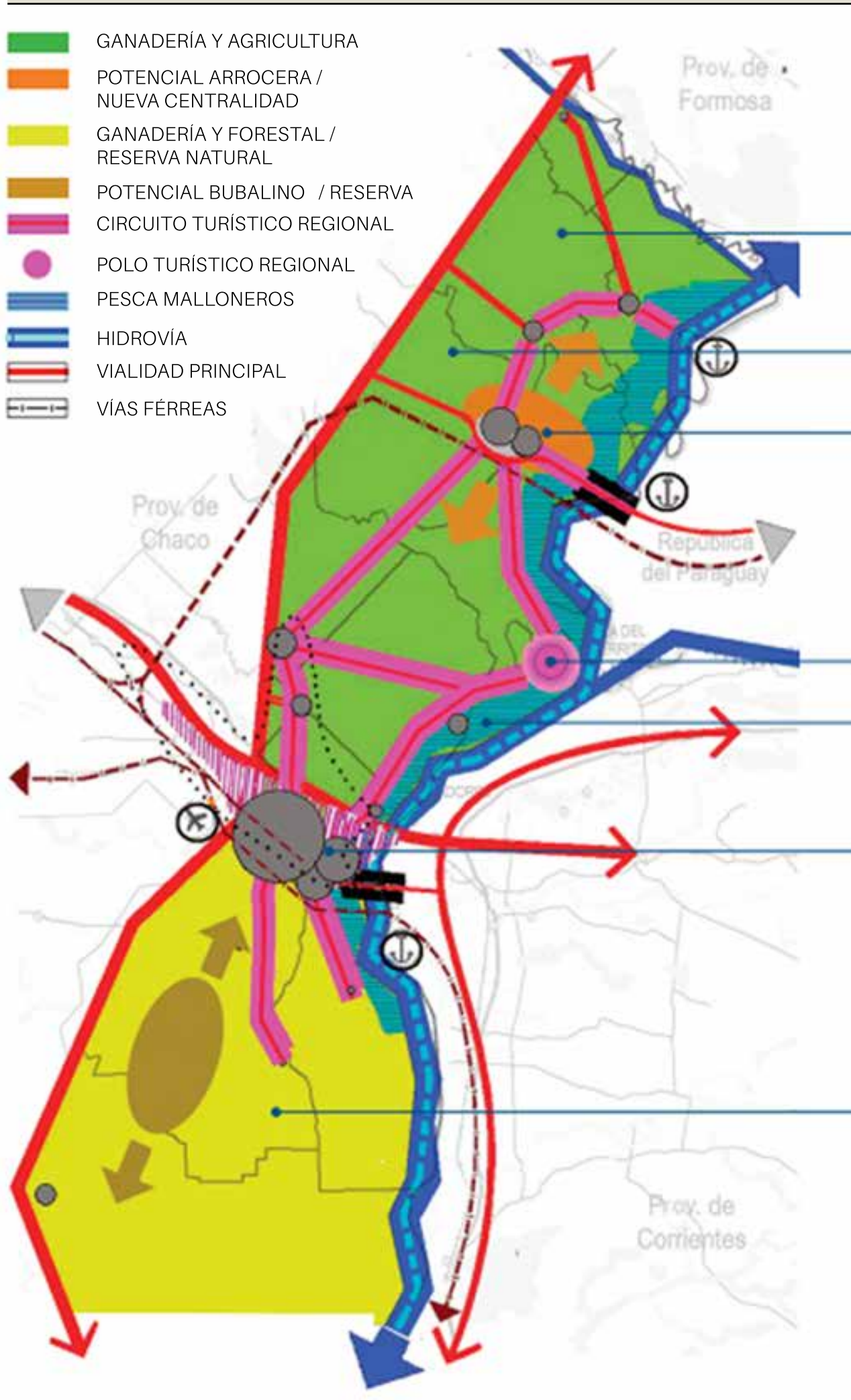




\section{Propuesta}

Propiciar el arraigo de la población en el Humedal Norte, a través del fomento de la expansión y diversificación productiva y del desarrollo del turismo, valorizando y preservando al mismo tiempo su patrimonio socio-productivo actual y la calidad ambiental que le otorga la categorla de Sitio RAMSAR.

Fortalecer el desarrollo y la cohesión del sistema de centros, mediante la inversión en la mejora de la red de caminos rurales que garantice la interconectividad y accesibilidad a los bienes y servicios que estos proveen, así como en la dotación de energía y conectividad digital en tanto factores determinantes de la productividad e inclusión social.

Fomentar la consolidación del nodo Las Palmas-La Leonesa como centralidad microrregional, estrechamente asociada con el potencial productivo y logístico que suponen el nuevo Puerto de Las Palmas y la vialidad proyectada para su acceso, así como con la perspectiva de integración regional sudamericana que implica el proyecto del puente internacional Las PalmasNanbecuy.

Fomentar un Circuito Turístico del Humedal, que potencie la oferta paisajística y patrimonial de cada localidad y consolide a la isla del Cerrito como polo de atracción regional, para sostener e impulsar la inversión pública y privada para la cualificación y ampliación de su oferta actual.

- Encaminar la reconversión productiva de la pesca artesanal, junto con el fomento de la acuicultura y de la pesca deportiva, en tanto actividades identitarias que forman parte del capital socio-productivo y cultural del humedal y que requieren politicas públicas estructurales de cara a la preservación de la fauna ictícola y a la mejora de la calidad de vida de sus habitantes.

Cosolidar al AMGR como polo logístico de servicios del nordeste argentino, gestionando la inversión en obras de alto impacto regional como la construcción del puente Chaco-Corrientes, la rehabilitación plena del ferrocarril Belgrano Norte y la consolidación operativa y de accesibilidad del puerto de Barranqueras.

Delimitar un área de reserva natural en el Humedal Sur, con un estricto control de las actividades productivas compatibles con la preservación de su riqueza paisajística y de biodiversidad -tal como la expansión de la ganadería bubalina-, junto con la inversión en infraestructuras de accesibilidad y equipamiento para el desarrollo del turismo ecológico. 
Estas características, asociadas a la existencia de grandes superficies de tierra fiscal, constituyen una fortaleza de cara a la preservación del ecosistema, así como a la posibilidad de su goce bajo condiciones de buen manejo ambiental.

Se propone, por tanto, la delimitación de un área de reserva natural con estricto control de las actividades productivas, de modo de garantizar la preservación del ecosistema, junto a la promoción del turismo sustentable. En segundo lugar - sin que suponga orden de prelación-elfomento a la expansión de la actividad ganadera bubalina, habida cuenta de su adaptabilidad a este medioambiente, de la existencia actual en la subregión de un núcleo productivo de alta eficiencia y de la potencialidad económica de la actividad, de la que la provincia de Chaco participa en términos regionales.

Por último, el sector norte, donde el humedal conjuga su potencial ambiental con buenas condiciones para el desarrollo de asentamientos humanos, concentra las mayores oportunidades para la producción agropecuaria, asociada a la expansión del turismo rural que habilita su riqueza paisajística. Un territorio donde radica la mayor cantidad de población vinculada con la ruralidad, con un evidente potencial de diversificación económica, merece particular atención para sostener el tejido social existente fomentando el arraigo. Esto es sinónimo de invertir en infraestructura, equipamiento y servicios que apalanquen la inversión privada para ampliar la oferta de empleo, que mejoren la calidad de vida de los habitantes y que viabilicen la cohesión territorial.
La apuesta superadora en este sentido es que logren cohesionarse como un circuito turístico que represente una verdadera oferta a nivel provincial y regional, lo que tiene como condición de factibilidad fundamental que se sostenga una red vial secundaria y terciaria que garantice la articulación de recorridos. Dentro de esta estrategia se destaca la vocación y potencialidades de la Isla del Cerrito.

\section{Estrategias, programas y proyectos de intervención territorial}

El conjunto de políticas públicas e iniciativas proyectuales planteadas como resultado del proceso de trabajo llevado a cabo en el marco del plan se han sintetizado mediante una Matriz de Estrategias, Programas y Proyectos de Intervención Territorial. Esta se estructura a partir de los tres grandes ejes estratégicos anteriormente mencionados, de los cuales derivan diez Programas de Actuación Territorial (PAT), y en relación con cada uno de estos, se identifican dieciséis Subprogramas de Actuación Territorial (SP). La matriz resultante da cuenta del marco conceptual con el que se abordó la fase propositiva del plan maestro, la que conjuga programas de promoción y fomento a la producción con programas de inversiones en capital fijo y programas de contenido netamente social o institucional. Por ello, dentro de esta estructura se sitúan 76 proyectos clasificados según se trate de propuestas para la ejecución de infraestructura y equipamiento o bien de consolidación de recursos sociales e institucionales. De este modo, la matriz representa un momento de una dinámica de trabajo desarrollada, que puedecompletarse con sucesivos proyectos a lo largo del tiempo, y su valor principal es el de aportar un marco conceptual que, partiendo de sólidos ejes estratégicos, formula programas de actuación territorial basados en lineamientos de acción ineludibles para el desarrollo armónico del humedal.

\section{Comentarios finales}

El libro del Plan Maestro Humedales fue presentado en julio de 2017. Cumpliendo con el objetivo desde el que fue planteado, se ha constituido en instrumento de gestión y orientación de la política pública en el territorio que abarca.

De la misma manera que se ha acompañado la elaboración del plan con acciones concretas en territorio, actualmente se gestionan los proyectos planteados para el barrio Pedro Pescador, continúan las obras en Isla del Cerrito para posicionar su rol turístico con cualidadesúnicas en la región, las obras en Puerto Las Palmas, que posicionará no solo a la provincia en la región y a nivel internacional.

Se elaboraron tres planes sectoriales que profundizan la mirada en territorios cuyas complejidades requieren un análisis pormenorizado. Constituye un desafío adoptar y mantener el uso de la matriz como instrumento adaptado a la dinámica de la gestión pública, conservando las premisas iniciales de garantizar la sustentabilidad del ecosistema junto con la mejora de los indicadores y condiciones socio-territoriales de la población residente. 